\title{
Logiques d'acteurs et marchés fonciers : une lecture transylvanienne (Roumanie)
}

\author{
Rozenn Trédan ${ }^{1, *}$ et Jean-Philippe Colin ${ }^{2}$ \\ ${ }^{1}$ Ingénieure en agro-développement de l'ISTOM, Cergy, France \\ 2 Directeur de recherche à l'IRD, UMR GRED, Montpellier, France
}

\begin{abstract}
Résumé - Cet article propose un décryptage des logiques d'acteurs dans leur rapport aux marchés fonciers de l'achat-vente et de la location, dans le contexte de la Transylvanie (Roumanie). L'analyse des pratiques foncières met en lumière un faible dynamisme de l'achat-vente, dû principalement à l'étroitesse de l'offre. Les propriétaires ne disposant pas des moyens d'exploiter directement leur terre, ou impliqués dans des activités hors agriculture, privilégient souvent sa cession en faire-valoir indirect (FVI) plutôt que la vente. La location assure en effet une rente régulière, susceptible de compléter les faibles revenus du ménage ou de contribuer à son alimentation, et le maintien de la terre dans le patrimoine familial, y compris comme un capital réalisable en cas de besoin. D'autres propriétaires se positionnent dans une logique de vente mais préfèrent céder en FVI en pariant sur une hausse attendue du prix de la terre. Les demandeurs potentiels sur le marché de l'achat-vente, en l'absence d'offre, sont souvent contraints de recourir à la prise en FVI. Ces acteurs sont majoritairement les sociétés commerciales et les exploitations familiales marchandes. Cette situation a conduit à l'émergence de configurations de tenure inversée qui s'inscrivent dans des dynamiques de concentration de terres à grande échelle.
\end{abstract}

Mots clés : marché foncier / achat-vente / faire-valoir indirect / Roumanie

\begin{abstract}
Actors' rationales and rural land markets: insights from Transylvania (Romania). This article explores actors' rationales regarding their participation in rural land sales and tenancy markets in the context of Transylvania (Romania). The analysis of land practices highlights the low dynamism of the land sales market, linked to a thin supply. The landowners potentially most likely to sell - those who lack the means to directly use their land, or are involved in off-farm activities - very often favor leasing out their land. This ensures them a regular source of income or product (in the case of in-kind leases), supplementing the household's often meager revenues, and maintains the land patrimony (including land property as a realizable asset in case of necessity). Other landowners plan to sell their land but prefer to rent it out on the short run, because of an expected increase in land prices. Facing a lack of supply on the sales market, the potential buyers of land have to lease in land. This is particularly the case of commercial companies, and of market-oriented family farms. This situation has led to the emergence of reverse tenancy configurations.
\end{abstract}

Keywords: land market / land sale / tenancy / Romania

\section{Introduction}

La question des marchés fonciers est devenue incontournable dans la problématique du développement rural, au Sud comme dans les pays «en transition » (Deininger et Feder, 2001). Dans le cas de ces derniers, Swinnen et Vranken (2007) identifient les faits suivants : (i) le marché de l'achat-vente est généralement nettement moins développé que celui du fairevaloir indirect (FVI) ; (ii) ces deux types de marchés sont caractérisés par des coûts de transaction importants ; (iii) le type de politique de décollectivisation mise en œuvre influe

\footnotetext{
$\overline{\text { *Auteur de correspondance }}$ : roztredan@gmail.com
}

directement sur la structure agraire qui émerge, et donc potentiellement sur la structure des marchés fonciers. Les politiques de restitution créent ainsi les conditions d'une offre (en particulier sur le marché du FVI) en réallouant les droits de propriété à des individus qui ne sont pas/plus actifs dans l'agriculture, et d'une demande, avec l'émergence d'une agriculture de type entrepreneuriale à grande échelle.

La Roumanie est à cet égard illustrative. Le pays a connu un régime communiste entre 1945 et 1989, avec alors plus de $90 \%$ des terres labourables collectivisées à travers des structures coopératives ou étatisées. La politique de décollectivisation, de restitution des terres aux anciens propriétaires (qui avaient conservé un droit de propriété purement formel sur 
leur terre sous le régime Ceausescu) et de dotation de lopins aux anciens coopérateurs sans terre, au début des années 1990, a induit une extrême fragmentation du parcellaire et une (ré) allocation des droits de propriété à une population globalement âgée et souvent sans rapport productif direct à l'agriculture ; selon Dumitru (2002), moins de $20 \%$ des bénéficiaires des restitutions/dotations avaient une activité agricole. La réorganisation d'une partie des anciennes coopératives sous forme de «sociétés agricoles » (Societatea agricola, SA) et la privatisation des domaines d'État et des stations de mécanisation sous la forme de «sociétés commerciales 》 (Societatea comerciala, SC) ont quant à elles permis le maintien ou le développement d'unités de production à grande échelle, sans base foncière propre pour certaines et accédant alors à la terre à travers le marché du FVI (Amblard, 2006). Les chiffres les plus récents montrent une polarisation de l'agriculture roumaine, avec plus de la moitié du nombre total d'exploitations qui exploitent moins d'un hectare et occupent $5 \%$ de la SAU, tandis que les exploitations supérieures à 100 ha représentent $0,35 \%$ du nombre total d'exploitations et occupent $49 \%$ de la SAU (INS, 2012).

Le marché de l'achat-vente apparaît comme relativement peu actif ; selon Dumitru et al. (2004), la surface agricole vendue entre 1999 et 2004 représenterait seulement $3 \%$ de la surface agricole. La lourdeur et la durée des démarches, les coûts de transaction, les contraintes d'accès au crédit, la fragmentation du parcellaire, la détention de terres en indivision par un grand nombre d'héritiers, peuvent expliquer ce faible dynamisme. À l'inverse, le marché du FVI serait en forte progression. Le dernier recensement agricole (INS, 2012) révèle qu'en 2010, $28 \%$ de la surface totale agricole était exploitée en FVI, essentiellement par des exploitations avec personnalité juridique (70\% des superficies en FVI). Ces chiffres sont loin de capturer le niveau réel de l'activité du marché, puisque de nombreuses transactions sont réalisées hors du système formel.

La présente étude a pour objectif d'identifier les logiques d'acteurs dans leurs rapports aux marchés fonciers ruraux dans un contexte roumain. Explorer ces logiques suppose de positionner les options et choix des acteurs dans des jeux d'alternatives potentielles : exploiter ou non directement la terre en faire-valoir direct (FVD), céder en FVI ou vendre la terre, prendre en FVI ou acheter de la terre, conserver la terre sans qu'elle soit exploitée. Notre modèle conceptuel élémentaire prend en compte :

- la fonction-objectif des acteurs : assurer une production (d'autoconsommation ou marchande) ou percevoir une rente foncière, spéculer (projet de vente de terre, mais report de la cession dans l'attente d'une hausse des prix), construire un patrimoine (logique de transfert intergénérationnel ou de sécurisation d'une production d'autoconsommation);

- l'intérêt comparatif, en termes de calcul économique réalisé dans la logique des acteurs, de la prise en FVI ou de l'achat, ou alternativement de la cession en FVI ou de la vente ;

- les dotations en facteurs, en tant que contraintes potentielles relativement à une exploitation en FVD et déterminants de cession en FVI de tout ou partie des terres disponibles, ou de vente, ou à l'inverse assurant une capacité de prise en FVI ou d'achat de terre ;

- les imperfections des marchés des facteurs, avec en particulier le rôle possible de la défaillance de dispositifs de crédit ou d'assurance (assurance maladie, décès, chômage, retraite) dans l'existence de ventes (ou de cession en location) de détresse ;

- les coûts de transaction sur les marchés du FVI et de l'achatvente (coût de recherche du partenaire, de regroupement de parcelles prises en FVI, etc.), ainsi que les coûts de défense des droits de propriété sur des parcelles non exploitées.

L'ensemble de ces éléments est à apprécier en fonction de la configuration de marché, définie comme la caractérisation schématique du positionnement socio-économique relatif des catégories des preneurs et des cédants sur les marchés fonciers.

\section{Démarche et production des données}

Nous avons opté pour une démarche compréhensive, i.e. visant à comprendre les pratiques d'acteurs en tenant compte de la perception que ces derniers ont de leur situation et des options qui leur sont offertes (Simon, 1986). Comme usuellement dans les démarches de recherche qualitative, nous avons eu pour objectif de produire une connaissance non pas représentative en termes statistiques ou géographiques, mais significative de situations et de pratiques foncières (Denzin et Lincoln, 2005).

Les enquêtes de terrain ont été menées dans le judet (département) Mures, en Transylvanie (essentiellement dans les villages de Ganesti, Bagaciu et Bogata). Son climat, son relief et ses types de sols en font une zone intermédiaire entre les zones de montagnes et les zones de grandes plaines. Les productions végétales et animales rencontrées dans les Mures sont très diversifiées et couvrent une majorité de situations rencontrées à l'échelle nationale. La zone a été collectivisée puis décollectivisée, ce qui est le cas de la majorité des terres du pays. Le cadastre n'est pas absent, même s'il est très incomplet, ce qui là encore représente une situation intermédiaire dans les cas identifiés en Roumanie. Enfin, on y retrouve tous types de structures, y compris quelques sociétés à capitaux étrangers.

Notre analyse repose sur un travail de recension bibliographique sur la question foncière en Roumanie, sur la collecte de documents administratifs (textes de loi, cartes, registres fonciers, extraits de cadastres, titres de propriété, contrats de fermage), et sur les enseignements tirés d'une immersion dans la zone d'étude et d'entretiens semi-directifs. Ces entretiens ont été conduits entre mars et septembre 2014 auprès de 22 acteurs institutionnels (ministère de l'Agriculture, mairies, Agence des domaines de l'État, etc.) et professionnels « support» (avocats, notaires, géomètres, etc.), et de 35 exploitants ou propriétaires fonciers (identifiés avec l'appui initial des mairies, puis dans une stratégie de boule de neige). Nous avons stratifié l'échantillon des exploitants ou propriétaires fonciers afin de couvrir une variation maximale de types d'acteurs au regard de notre objet d'étude, avec la recherche d'un gradient de cédants/vendeurs effectifs ou potentiels (groupes 0 à 2) à preneurs/acheteurs effectifs ou potentiels (groupes 3 à 7) (Tab. 1).

De nombreux acteurs ont été visités à plusieurs reprises, avec de longs entretiens (le temps de visiter les terres et l'exploitation, de rencontrer la famille, etc.). Ces entretiens ont été menés avec une interprète, étudiante en sociologie.

$\mathrm{Du}$ fait des orientations méthodologiques retenues pour cette étude, ces enquêtes ne seront pas mobilisées comme base 
Tableau 1. Exploitants et/ou propriétaires fonciers enquêtés.

Table 1. Farmers and/or landowners surveyed.

\begin{tabular}{|c|c|c|c|c|c|}
\hline Types d'acteurs enquêtés & Nombre & \multicolumn{4}{|c|}{ dont concernés par } \\
\hline Propriétaire cédant toutes ses terres en FVI & 5 & 5 & 2 & 5 & 3 \\
\hline Exploitation minifundiste $(<5$ ha $)$ & 4 & & 1 & 3 & \\
\hline Exploitation familiale moyenne (5-30 ha) & 5 & & 2 & & 2 \\
\hline Société commerciale $<200$ ha & 5 & & 3 & & 5 \\
\hline Société commerciale $>200$ ha & 5 & & 2 & & 5 \\
\hline Société commerciale à capitaux étrangers & 4 & & 2 & & 4 \\
\hline Total & 35 & 7 & 25 & 10 & 27 \\
\hline
\end{tabular}

Chiffres en gras : pratiques antérieures aux entretiens. Un type d'acteur pouvant avoir eu recours à plusieurs pratiques au cours du temps, les totaux peuvent être supérieurs au nombre d'enquêtés. FVI : faire-valoir indirect.

de quantification (elles ne visaient pas la représentativité statistique), mais pour apprécier les logiques d'acteurs dans leurs rapports aux marchés fonciers.

\section{Le rapport aux marchés fonciers: une offre privilégiant le FVI plutôt que la vente}

S'interroger sur les logiques d'acteurs dans leur rapport à l'offre sur les marchés fonciers demande d'explorer les alternatives que sont l'exploitation directe, la vente, la nonmise en valeur de la terre possédée et la cession en FVI. Cette dernière peut s'opérer avec : (i) des contrats de location (rente fixe versée en nature ou en numéraire - au moment de l'étude entre 0,7 et 1 tonne de blé/ha/an ou entre 560 et 800 lei/ha/an, soit 125-182 euros), selon le pouvoir de négociation des parties, la capacité financière du preneur, les qualités de la parcelle et les rapports entre les acteurs, la rente étant plus élevée lorsque le preneur est une société à capitaux étrangers ; (ii) des contrats de métayage avec ou sans implication du propriétaire dans la production; (iii) des cessions de terres à des sociétés agricoles, en tant que sociétaires de ces dernières (voir Amblard, 2006).

À travers nos enquêtes, la vente de terre intervient dans trois types de situation :

- il peut s'agir de ventes de détresse, en cas de choc (maladie, chômage dans le cas de pluriactifs, etc.). La vente n'est alors pas souhaitée, mais contrainte ; elle conduit à se séparer progressivement ou en une fois de la totalité ou d'une partie des terres. Cette logique est à mettre en rapport avec l'absence de dispositif d'assurance et les difficultés d'accès au crédit pour les petits exploitants/propriétaires et de faibles niveaux de revenus;

- la vente peut être liée à des difficultés lors de la recherche d'un locataire, lorsque le propriétaire ne souhaite pas ou ne peut pas assurer une production en FVD (propriétaire « passif »). Il s'agira en particulier de parcelles difficiles d'accès, de très petite superficie, en pente ou dont les sols ne sont pas de bonne qualité. Intervient également le fait que les preneurs potentiels sont peu nombreux. Nous verrons que la majorité des petits et moyens exploitants agricoles sont peu actifs en tant que preneurs sur le marché du FVI (Tab. 1). Lorsqu'ils ne sont pas directement sollicités par un tenancier, les propriétaires doivent rechercher eux-mêmes un partenaire, ce qui suppose une bonne insertion dans les réseaux locaux. De surcroît, les parcelles qui ne sont pas exploitées doivent être surveillées - lors de nos entretiens, plusieurs cas d'appropriation indue de ce type de parcelles ont été documentés. La difficulté à trouver un tenancier et les coûts de défense des droits sur des terres non exploitées expliquent que des propriétaires " passifs » finissent par vendre leur terre - lorsqu'un acheteur se présente ;

- certains acteurs font le choix de vendre leur terre, du fait de leur fonction-objectif. C'est le cas de propriétaires urbains ou émigrés ayant hérité de terre, n'ayant pas d'intérêt pour l'agriculture, retraités ou engagés dans une activité professionnelle autre qu'agricole, qui du fait de leur éloignement géographique et/ou de leur désintérêt pour l'agriculture, ne trouvent pas d'avantage à conserver leurs terres en propriétés.

L'élément fort qui ressort cependant de nos investigations reste le caractère peu dynamique du marché de l'achat-vente, du fait d'une faiblesse de l'offre, les propriétaires n'exploitant pas en FVD optant plutôt pour la cession en FVI que pour la vente. Cette faiblesse de l'offre est à mettre en rapport avec plusieurs facteurs :

- la propriété foncière joue un rôle essentiel pour les producteurs de subsistance. Elle leur permet de bénéficier de l'intégralité de la production, ce qui est important lorsque de petites surfaces sont exploitées pour l'autoconsommation, et apporte une sécurité d'accès à la terre ;

- la terre représente un dispositif d'assurance mobilisable (en dernier recours) en cas de choc ;

- la terre est un patrimoine potentiellement transmissible aux héritiers, et la propriété foncière revêt une dimension symbolique, affective (Gaborean, 2011), qui tend à s'atténuer avec la nouvelle génération d'héritiers mais 
qui est toujours présente chez les propriétaires enquêtés, hors sociétés commerciales pour qui la propriété ne revêt aucune représentation symbolique ;

- la propriété foncière est une source de rente, en espèce ou en nature - contribuant alors directement à l'alimentation du ménage du cédant ou de son bétail. Les propriétaires rencontrés concernés par la cession en FVI évoquent l'avantage d'un fermage à échéance régulière, comparativement à une entrée d'argent unique et importante lors d'une vente, qui peut se retrouver rapidement dilapidée ;

- la vente peut être reportée (et la cession en FVI privilégiée à court terme) dans l'attente d'une augmentation du prix de la terre. Cette logique, exprimée lors de nos entretiens, est tout particulièrement le fait de propriétaires urbains ou émigrés ayant hérité de terre, n'ayant pas d'intérêt pour l'agriculture, retraités ou engagés dans une activité professionnelle autre qu'agricole. Elle est à mettre en rapport avec l'arrivée d'investisseurs (roumains ou étrangers) dans l'agriculture et l'entrée dans l'Union Européenne (voir Swinnen et Vranken, 2007, pour un constat similaire au niveau plus général des pays en transition intégrant l'U.E.). Plus récemment, c'est la loi 17/2014 réglementant l'achat/vente de terre en Roumanie qui renforce ce phénomène de rétention.

La cession en FVI, alternativement à la production en FVD, relève de deux types de logique :

- elle peut correspondre à une stratégie rentière, lorsque la fonction-objectif n'est pas - ou plus - la production : propriétaires urbains ou émigrés ayant hérité de terre, n'ayant pas d'intérêt pour l'agriculture, retraités ou engagés dans une activité professionnelle autre qu'agricole ;

- elle peut aussi venir de contraintes sur les facteurs de production (travail, capital, trésorerie), pour des acteurs qui, s'ils le pouvaient, exploiteraient en FVD toutes leurs disponibilités foncières. Ce sera en particulier le cas d'anciens agriculteurs qui ne sont plus en capacité de travailler, notamment du fait d'un âge avancé, d'un manque de moyens de production, de l'éloignement des parcelles ou encore d'un déficit en main d'œuvre (absence de maind'œuvre familiale ou difficulté à recruter une main-d'œuvre extérieure), et qui cèdent en FVI toute la terre qu'ils possèdent. Ce sera également le cas de certains exploitants de subsistance, qui conservent en FVD une petite parcelle et cèdent le reste en FVI.

\section{Le rapport aux marchés fonciers : une demande privilégiant l'achat mais souvent contrainte à la prise en FVI}

La demande sur les marchés fonciers est à interpréter au regard de l'alternative prise en $\mathrm{FVI} /$ achat.

En premier lieu, la prise en FVI peut venir de contraintes sur l'achat de terre, qui serait sinon privilégié par les acteurs :

- ces contraintes à l'achat renvoient d'abord à la faiblesse de l'offre, que nous venons d'évoquer. Les acteurs souhaitant élargir leur patrimoine foncier peinent à trouver des vendeurs d'une manière générale, ou du moins sur les terres qu'ils souhaitent acheter, i.e. celles adjacentes aux parcelles déjà exploitées ;

- le prix du foncier, en forte augmentation depuis une dizaine d'années (il n'existe pas de statistiques officielles sur l'évolution des prix de la terre en Roumanie, mais les données recueillies auprès de cabinets d'avocats et d'études de notaires ainsi que l'ensemble des discours recueillis vont dans le même sens), exclut les acteurs les moins dotés en capacités de financement. De surcroît, les coûts de formalisation des transactions sont très élevés : ils représentent en moyenne $33 \%$ du prix de la terre, pour l'acheteur ;

- le morcellement extrême de la propriété crée des coûts de transaction considérables dans leur composante « coûts de recherche d'un partenaire ». On observe ainsi des situations où un acheteur doit traiter avec une centaine de propriétaires pour reconstituer une parcelle de 10 ha d'un seul tenant.

Les exploitations commerciales, mieux dotées en capacités de financement, se heurtent avant tout au premier de ces facteurs. L'ensemble de ces facteurs jouent pour les exploitations familiales désireuses de s'agrandir par l'achat.

En second lieu, la prise en FVI, alternativement à l'achat, peut être un choix, exprimé avant tout par des responsables de sociétés commerciales :

- la prise en FVI permet de ne pas immobiliser du capital dans la terre, et de l'investir plutôt dans le matériel, les infrastructures et les intrants ;

- les termes des contrats de FVI favorisent largement les sociétés, leur pouvoir de négociation étant nettement supérieur à celui des bailleurs. Nous avons également relevé de nombreux cas de contrats conclus avec une forte asymétrie d'information, le cédant n'ayant pas compris ou n'étant pas au courant de certains termes du contrat (voir également Amblard et Colin, 2009). Les sociétés commerciales obtiennent ainsi des conditions qu'elles estiment avantageuses, notamment une rente en nature relativement faible comparée aux rendements - les rendements en céréales observés lors de nos enquêtes étant près de deux fois supérieurs pour les sociétés commerciales que pour les exploitations familiales; la rente, qui représente près de $22 \%$ du rendement pour ces dernières, n'atteint que $12 \%$ pour les sociétés commerciales.

Les exploitants familiaux rencontrés lors de l'étude ont tous évoqué le souhait d'acheter les terres qu'ils prennent aujourd'hui en FVI, afin de sécuriser leur accès au foncier (ne plus dépendre du marché du FVI), mais aussi d'agrandir leur patrimoine, transmissible à des héritiers. L'accès à la propriété, comparativement à la prise en FVI, est également vu comme un filet de sécurité, un capital mobilisable en cas de besoin. Ils souhaitent ainsi que la location ne soit qu'une étape, et tous attendent que les propriétaires se décident à vendre, même s'ils évoquent, pour la plupart, la difficulté qu'ils auraient pour financer l'achat.

Les sociétés commerciales, qui exploitent essentiellement des terres prises en location (plus de $75 \%$ de la surface totale des sociétés enquêtées, conduisant à des configurations de tenure inversée) sont de plus en plus demandeuses sur le marché de l'achat/vente - par tenure inversée, on entend des 
situations dans lesquelles des petits propriétaires cèdent en FVI une partie ou la totalité de leurs disponibilités foncières à de (plus) grands propriétaires fonciers ou à des entrepreneurs agricoles qui, sans toujours posséder de terre, disposent du capital d'exploitation, d'un accès au capital financier et de compétences techno-économiques et de capacités organisationnelles (Amblard et Colin, 2009 ; Colin, 2014). Les coûts induits par la recherche de propriétaires, la conclusion des contrats de location et leur renouvellement, et le paiement de la rente (souvent en nature, au domicile du propriétaire) sont considérés par les responsables de ces sociétés comme supérieurs aux coûts de transaction liés à l'achat d'une terre, lorsque les surfaces cultivées sont importantes (un ou plusieurs employés doivent être spécialement mobilisés pour ces tâches) - à titre d'illustration, une société commerciale enquêtée a dû établir 764 contrats pour 630 ha pris en location. De plus, les sociétés commerciales subissent une relative insécurité juridique sur les contrats de location : doublons de contrats (les mairies peuvent parfois valider deux contrats de location sur une même parcelle), surfaces théoriquement louées qui ne correspondent pas à la réalité, risque de non-renouvellement de contrat, en particulier lorsque le propriétaire décède. Dans ce dernier cas, la recherche des héritiers est souvent complexe et donne lieu à des confusions quant au paiement de la rente (la législation roumaine oblige le tenancier à diviser le montant de la rente en parts égales entre chaque héritier). Cette insécurité entraîne de nombreux conflits fonciers (22 cas actuels ou passés évoqués lors de nos enquêtes), avec parfois une saisine de la justice (uniquement mentionnée par les sociétés commerciales, 9 cas), ce qui est onéreux, long et aléatoire. Les sociétés commerciales voient ainsi de plus en plus la prise en FVI comme une stratégie permettant à terme d'acheter les terres - la loi 17/2014 oblige les propriétaires à informer le locataire en cas de vente, ce dernier bénéficiant d'un droit de préemption. Les exploitations familiales bénéficient de moindres coûts de recherche que les sociétés commerciales sur le marché du FVI (interconnaissance des acteurs locaux), mais elles subissent proportionnellement davantage la contrainte du morcellement des parcelles (du fait d'un manque d'équipement). Les contrats étant généralement informels, à la différence des contrats conclus par les sociétés commerciales, il est plus difficile pour le preneur de faire valoir ses droits en cas de conflit et il ne peut pas faire jouer son droit de préemption en cas de vente de la parcelle.

\section{Conclusion}

L'analyse des pratiques foncières met en lumière un faible dynamisme de l'achat-vente, dû principalement à l'étroitesse de l'offre. Les propriétaires a priori les plus susceptibles de vendre - les propriétaires « passifs »-privilégient souvent la cession de leur terre en FVI. Cette dernière assure une rente régulière et le maintien des terres dans le patrimoine familial. Les ventes de terre permettent de répondre aux situations de détresse, et n'ont lieu qu'en dernier recours. Ainsi, les demandeurs potentiels sur le marché de l'achat-vente, en l'absence d'offre, sont souvent contraints de recourir à la prise en FVI. Ces acteurs sont essentiellement les sociétés commerciales (à capitaux étrangers aussi bien que nationaux) et les exploitations familiales marchandes. Cette situation a conduit à l'émergence de configurations de tenure inversée qui s'inscrivent dans des dynamiques de concentration de terres à grande échelle. Les sociétés commerciales sont en mesure d'imposer les types et les termes des contrats de FVI, et elles possèdent par ailleurs souvent une capacité financière qui leur permet de proposer des prix à l'achat que les exploitations familiales ne peuvent pas concurrencer.

Le $1^{\text {er }}$ janvier 2014 a été promulguée une nouvelle loi foncière réglementant le marché de l'achat-vente. Cette loi (17/ 2014) autorise désormais l'achat de terres par des citoyens et personnes morales de l'Union européenne - la location de terres par des personnes physiques et morales étrangères étant quant à elle autorisée depuis la loi 247/2005. Les effets à attendre de cette loi seront analysés dans une prochaine publication. On notera ici que, dans le contexte esquissé dans cet article, sa mise en œuvre ne vient pas perturber en profondeur les dynamiques en cours, mais devrait plutôt les entretenir, voire les renforcer. Elle ne facilite pas une ouverture du marché de l'achat-vente (du fait de la lourdeur des procédures prévues pour les transactions), et favorise clairement les exploitations les mieux dotées en capital financier et humain, celles-là mêmes qui sont actuellement les acteurs des concentrations à grande échelle de la propriété et/ou de l'exploitation. Le risque est donc d'observer un creusement de l'écart existant entre les sociétés commerciales et les exploitations familiales, laissant ces dernières à la marge, et les excluant progressivement de toute possibilité de développement. Cette tendance à la concentration foncière via le marché du FVI n'est par ailleurs pas réservée seule à la Roumanie, et peut être observée dans d'autres contextes similaires d'Europe, comme par exemple en Slovaquie et en République Tchèque (Swinnen et Vranken, 2007).

Remerciements. Ce texte fait suite à un stage de $\mathrm{R}$. Trédan au sein de l'ambassade de France en Roumanie. Il ne reflète en aucun cas la position de l'ambassade ou du gouvernement français. Nous remercions Sévrine Jacobs et Marie-Luce Ghib pour leur appui tout au long de l'étude, Oana Calen, interprète, ainsi que Valérie VilleminCiolos et trois lecteurs anonymes pour leurs commentaires sur des versions antérieures de ce texte.

\section{Références}

Amblard L. 2006. Les pratiques contractuelles foncières dans une économie en transition: le cas du département d'Alba en Roumanie. Thèse de doctorat en agro-économie, ENSA (Montpellier, France). http://prodinra.inra.fr/record/14000.

Amblard L, Colin J.-P. 2009. Reverse tenancy in Romania: Actors' rationales and equity outcomes. Land Use Policy 26(3): 828-836.

Colin J.-P. 2014. La concentration foncière par la tenure inversée (reverse tenancy). Etudes Rurales 194: 203-218.

Deininger K, Feder G. 2001. Land institutions and land markets. In: Gardner R, ed. Handbook of agricultural economics, vol. 1A. Agricultural Production. North Holland: Elsevier, pp. 288-324.

Denzin N, Lincoln Y, eds. 2005. The Sage handbook of qualitative research. Thousand Oaks: Sage.

Dumitru M. 2002. Romania-Country case studies on integrating land issues into the broader development agenda. Report commissioned by the World Bank, Bucharest (Romania).

Dumitru M, Diminescu D, Lazea V. 2004. Dezvoltare rurala si reforma agriculturii Romanesti. Bucarest: CEROPE. 
Gaborean F. 2011. Décollectivisation et relations de propriété dans un village roumain. Econ. Rural. 5/2011(n $\left.{ }^{\circ} 325-326\right)$ : 114-128.

INS. 2012. Recensement agricole de Roumanie. Institutul National de Statistică (INS), 2012. Recensaminte General Agricol 2010.
Simon H. 1986. Rationality in psychology and economics. J. Bus. 59 (4): S209-S224.

Swinnen J, Vranken L. 2007. Patterns of land markets developments in transition. Leuven: Katholieke Universiteit Leuven.

Cite this article as: Trédan R, Colin JP. 2016. Logiques d'acteurs et marchés fonciers : une lecture transylvanienne (Roumanie). Cah. Agric. 25: 25005. 